\title{
Sustained Stable Disease with Capecitabine plus Bevacizumab in Metastatic Appendiceal Adenocarcinoma: A Case Report
}

\author{
David Arias Ron ${ }^{a} \quad$ Carmen M. Labandeira ${ }^{b}$ Soledad Cameselle García ${ }^{a}$ \\ Jesús García Mata ${ }^{a}$ Mercedes Salgado Fernández ${ }^{a}$ \\ aMedical Oncology Department, University Hospital Complex of Ourense, Ourense, Spain; \\ ${ }^{b}$ Neurology Department, University Hospital Complex of Vigo, Hospital Alvaro Cunqueiro, \\ Pontevedra, Spain
}

\section{Keywords}

Appendiceal adenocarcinoma $\cdot$ Capecitabine $\cdot$ Bevacizumab $\cdot$ Stable disease

\begin{abstract}
In a patient who had been diagnosed in 2006 with appendiceal adenocarcinoma with peritoneal metastases after an incomplete surgery, palliative chemotherapy was administered. Firstline treatment with 5-fluorouracil, leucovorin and oxaliplatin (FOLFOX) and second-line treatment including 5 -fluorouracil, leucovorin and irinotecan (FOLFIRI) plus panitumumab showed inefficiency in controlling disease progression. Third-line chemotherapy combining capecitabine plus bevacizumab was started, achieving good control of the tumour growth and a minor response in the second computed tomography scan. We decided to maintain the treatment, although forced bevacizumab "breaks" were necessary due to unexpected adverse events, with the patient suffering disease progression every time bevacizumab was stopped and reaching minor response again once the antiangiogenic treatment was reintroduced. During more than 10 years after starting third-line treatment, the patient maintained good performance status and disease stability with this "up and down" management until January 2019, when a neurological adverse event during bevacizumab infusion drove us to abandon it definitely.

\section{Introduction}

Appendiceal malignancies are a group of rare tumours, of which the most frequent is mucinous adenocarcinoma (approximately 38\%), also including carcinoids (28\%), adenocarcinoma-not otherwise specified (27\%), and signet ring cell adenocarcinoma (7\%). The inci- 
dence of these tumours has increased by $54 \%$ during the past decade, reaching 0.97 cases per 100,000 population in the USA, probably indirectly related to the higher use of computed tomography (CT) scan that allows early diagnosis in asymptomatic patients [1]. In spite of that, the majority of patients are diagnosed with advanced disease (74\%), equally locally or with distant metastases. Similar results were observed by van den Heuvel et al. [2], who observed an increase in the incidence in the last decades, especially in women, and again mucinous adenocarcinoma being the one with the highest prevalence (48\%), pointing to an increased incidence of mucinous adenocarcinoma in men (1.0 per million person-years) and women (1.9 per million person-years). Diagnosis is usually made by abdominal imaging studies (CT scan) or as an incidental finding in abdominal surgery, with subsequent pathological confirmation.

Usually the treatment for localized appendiceal tumour is right hemicolectomy, but there is no available data analyzing the results of complementary chemotherapy or the role of radiotherapy. We, therefore, routinely transfer the results obtained in located colorectal cancer, treatment with chemotherapy based on 5-fluorouracil being the most commonly used adjuvant therapy [3]. Due to the small number of patients and the difficulty of performing randomized trials, there are no specific clinical practice guidelines for the metastatic presentation of this rare type of tumours. In most cases, the therapy is based on debulking surgery if it is feasible, considering the option of intraperitoneal chemotherapy in case of only peritoneal disease. The options of systemic chemotherapy, if necessary, are extrapolated from those used in metastatic colorectal cancer [4]. Due to this fact, available data on response to treatment, disease control, and long surveillance in this area are scarce. Here we present the case of a patient diagnosed with an appendiceal adenocarcinoma with peritoneal extension at diagnosis, who presented long disease stabilization with a third-line chemotherapy based on capecitabine combined with bevacizumab, showing high sensitivity to antiangiogenic treatment for more than a decade.

\section{Case Report/Case Presentation}

In May 2006, a 59-year-old man with hypertension and no other comorbidities underwent surgery for a right inguinal hernia, where a nodule in the hernial sac was found described as pseudomyxoma peritonei by the pathologist. A complete CT scan and colonoscopy were made after surgery, showing a filling defect with thickening of the caecum wall of approximately $5 \mathrm{~cm}$ of longitudinal extension, compatible with a primary tumour. Right hemicolectomy was decided on by the Tumour Board, but during the surgical procedure several myxoid masses were found at the appendix, minor omentum, pancreatic tail, and splenic cells. The surgery team resected the entire visible tumour, performing a right hemicolectomy with splenectomy, resection of the urachus and the greater omentum, but persisting unresectable peritoneal implants. The pathological diagnosis of the lesions after incomplete surgery was consistent with a well-differentiated appendiceal mucinous cystadenocarcinoma.

After the diagnosis of unresectable appendiceal cystadenocarcinoma was made, the patient was referred to the Oncology Department in July 2006, where he started first-line treatment with FOLFOX. He received 16 cycles, with minor disease response in the first CT control scan as best achievement, but in April 2007 several peritoneal implants increased in size, confirming disease progression by RECIST criteria (Fig. 1). We opted for a second-line treatment with FOLFIRI, where the epidermal growth factor receptor inhibitor panitumumab was added to clinical trial inclusion data (independent of RAS status) [5]. Minor disease response was again achieved in the first control CT scan, with stable disease during the following 18 months, but in November 2008 we observed several implants at the perihepatic, 

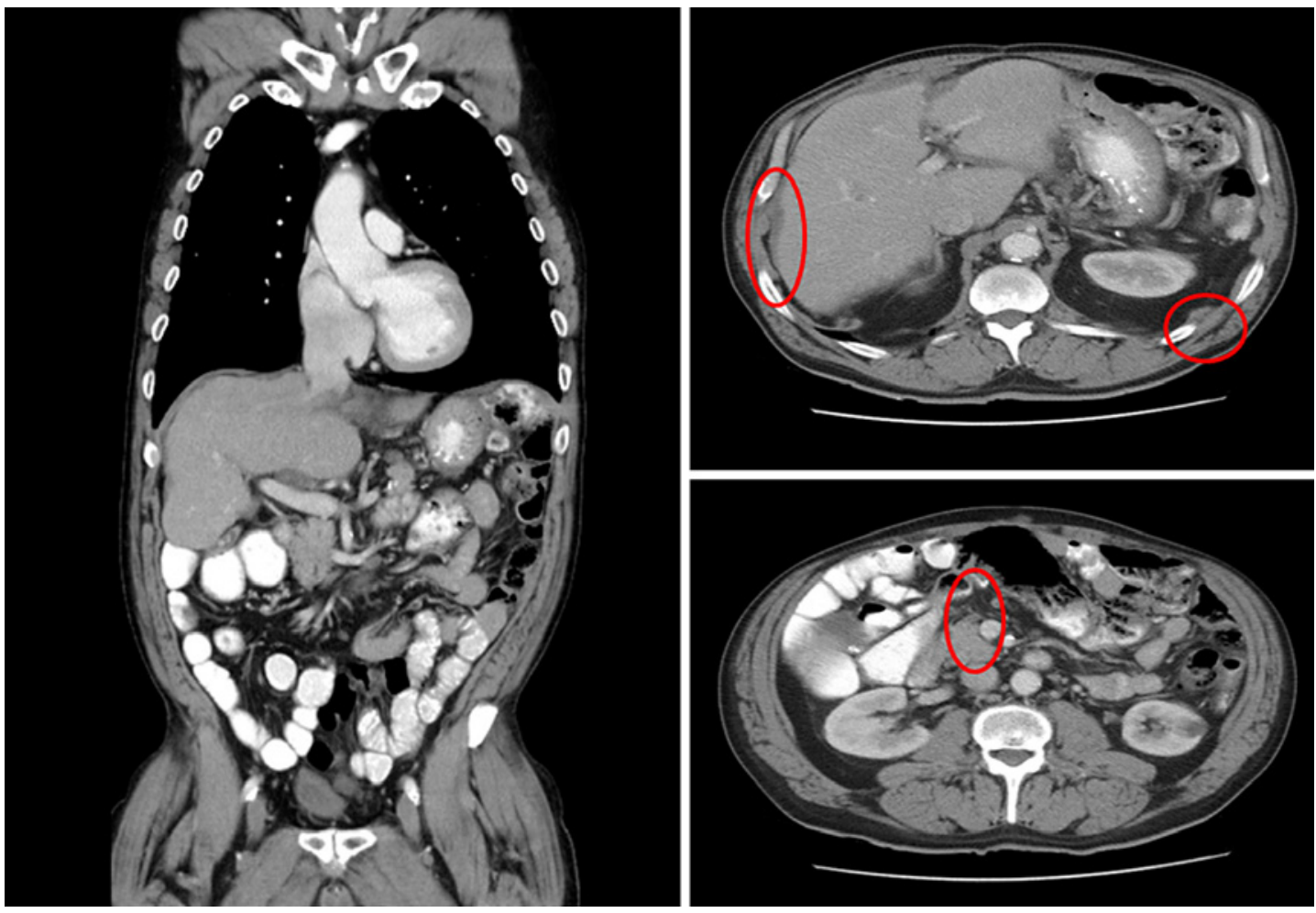

Fig. 1. CT scan after 16 FOLFOX cycles: the radiologist confirmed a negative response with an apparent increase in size of the intraperitoneal implants that predominate in the perihepatic area, hepatorenal space, left subphrenic space, a left lower hemiabdomen implant persisting in front of the psoas.

left subphrenic, and left hemiabdomen level, close to the psoas muscle, not present in previous image tests.

Considering the patient maintained a good performance status despite tumour progression, we decided to try a third-line of chemotherapy including capecitabine combined with the antiangiogenic bevacizumab. The patient started treatment in December 2008, reaching partial response during the first 6 months and later stable disease. Since the disease was located only at the peritoneal level, a PET-CT was performed confirming uptake of all peritoneal lesions, a surgical option was proposed with peritonectomy and hyperthermic intraperitoneal chemotherapy, but this was refused by the patient.

During third-line treatment, we were forced to temporarily stop using bevacizumab 3 times. The first occasion was in March 2010 due to a persistent grade 3 high blood pressure secondary to antiangiogenic treatment, not controlled with amlodipine plus enalapril and leading to stopping bevacizumab for 4 cycles. During this time, we confirmed with a CT scan disease progression at the peritoneal level, and we reintroduced bevacizumab once hypertension was corrected, achieving partial response in the first CT scan and stable disease during the next 5 years. The second situation was in February 2015 due to a dental procedure, when the patient received capecitabine monotherapy for 3 months; in the next CT, growth of all the perihepatic implants and appearance of at least two at the omentum were observed. We reintroduced bevacizumab obtaining again a partial response in the imaging studies. Finally, in September 2016 and at the request of the patient (he was not able to come to our centre to receive bevacizumab for several months), treatment was adjusted to capecitabine monotherapy, once again confirming progression of the implants after 5 months and observing 

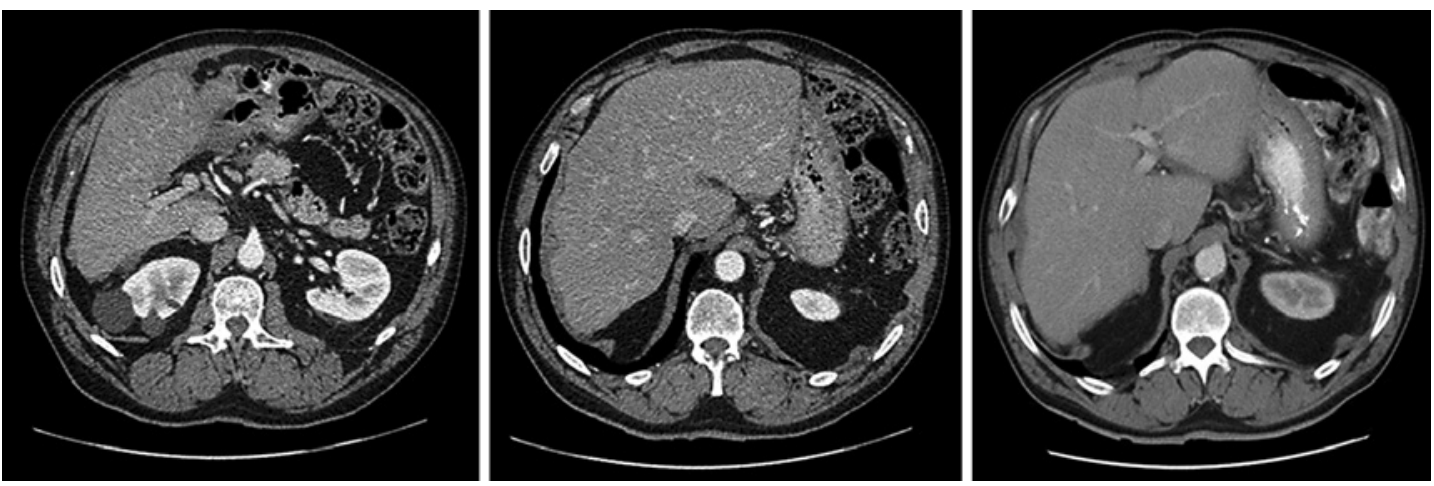

Fig. 2. CT scan in April 2017: stable disease of the intraperitoneal implants in the perihepatic area and in front of the left psoas, with no evidence of new malignant lesions after 9 years of capecitabine plus bevacizumab.

disease reduction after the reintroduction of the antiangiogenic treatment (Fig. 2). The treatment was well tolerated and no severe adverse events were observed during these years with the exception of the previously mentioned high blood pressure.

In September 2018, an intravascular catheter-related infection caused by methicillinresistant Staphylococcus aureus forced us to stop chemotherapy for 8 weeks because of intravenous antibiotic therapy and catheter removal. In the second cycle after bevacizumab reintroduction, the patient immediately suffered headache, dizziness, and right hemiparesis with numbness; these severe acute neurological signs appeared after administration of $10 \mathrm{~mL}$ of bevacizumab, showing complete recovery after stopping bevacizumab and administering 80 mg of intravenous hydrocortisone, and with an unremarkable brain CT scan. Considering the high risk of new neurological compromise, we decided to definitely stop capecitabine plus bevacizumab and started a fourth-line treatment with irinotecan plus cetuximab in January 2019. Unfortunately, the patient presented severe toxicity (grade 2 asthenia, grade 2 nausea and vomiting, and grade 3 mucositis) with tumour marker elevation; in May 2019 we, therefore, decided to start a new chemotherapy line with trifluridine/tipiracil treatment which is currently being maintained (Fig. 3).

\section{Discussion/Conclusion}

The recombinant, humanized monoclonal antibody bevacizumab plays an important role in angiogenesis inhibition by blocking the vascular endothelial growth factor (VEGF) and so tumour growth, proliferation, and invasiveness. In case of colorectal cancer, several phase III trials have shown the utility in terms of progression-free survival (PFS) and/or overall survival (OS) of bevacizumab plus chemotherapy in metastatic patients. First-line treatment in combination with oxaliplatin-based chemotherapy [6] and also bevacizumab addition to FOLFOX after progression to a first-line regimen without bevacizumab [7] reinforce the use of monoclonal antibody combined with fluoropyrimidine regimens. For example, $Q u$ et al. [8]analyzed the role of bevacizumab in this setting in a meta-analysis including nine clinical trials, showing strong positive data in terms of PFS (hazard ratio [HR]: 0.617; 95\% CI: 0.5300.719 ) and OS (HR $=0.848,95 \% \mathrm{CI}: 0.747-0.963$ ) and favouring the bevacizumab group; they describe the following already known adverse events: proteinuria, bleeding, hypertension, and thrombosis. Third- or later-line treatment including bevacizumab is less frequently 


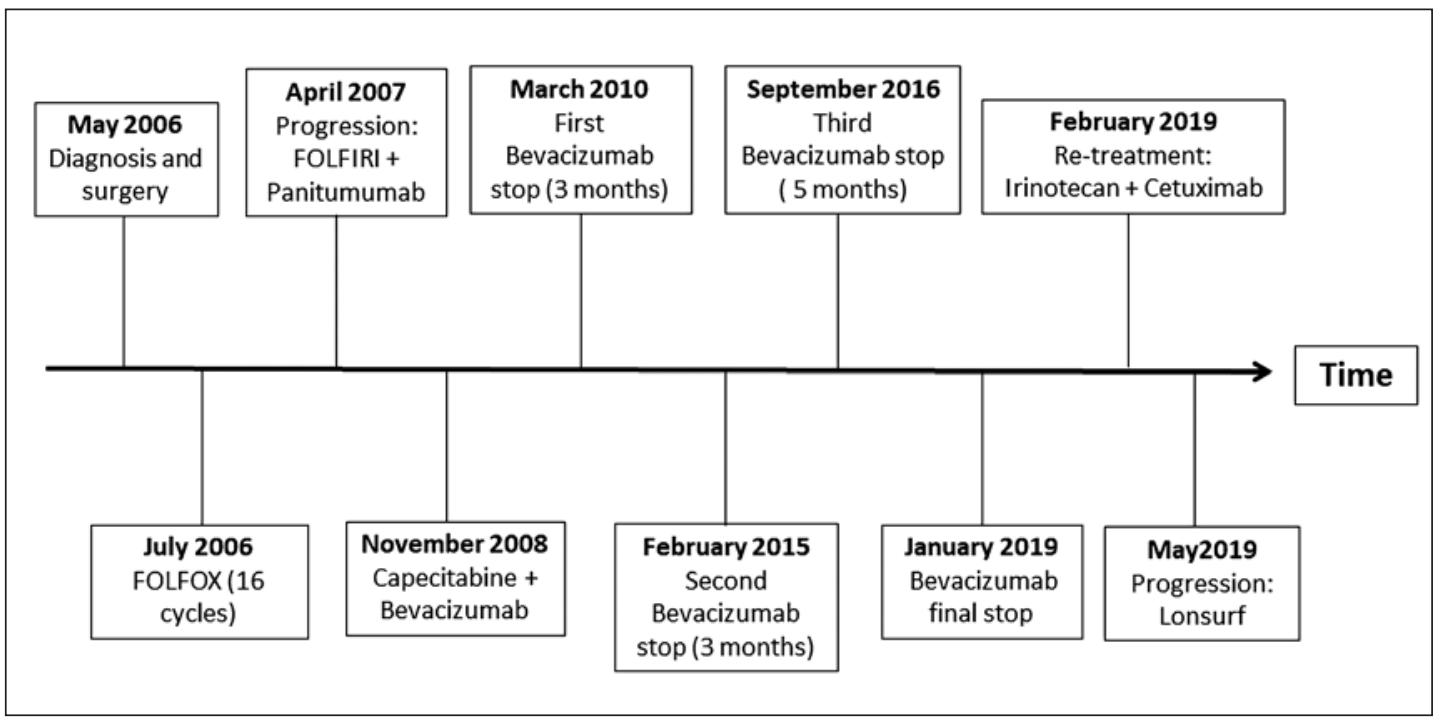

Fig. 3. Timeline of events since the diagnosis and a summary of administered treatments.

described in clinical practise, but several data suggest that combined regimens of bevacizumab plus chemotherapy could increase survival and achieve an acceptable disease control, with a PFS of 5.98 months and an OS of 14.77 months in a retrospective analysis [9].

The combination of capecitabine plus bevacizumab in metastatic colorectal cancer has also been tested in several trials, achieving particularly interesting results in the maintenance setting. The CAIRO3 trial showed a significant benefit for maintenance treatment versus observation when analyzing time from randomization to progression after chemotherapy reintroduction (PFS2, 11.7 vs. 8.5 months, HR 0.63) [10]. Unfortunately, data from capecitabine combined with monoclonal antibody in third or successive lines is scarce. Larsen et al. [11] endorsed the use of this combination in 34 metastatic colorectal cancer patients who had progressed to previous treatments with oxaliplatin, irinotecan, fluoropyrimidines, and anti-EGFR treatment in two or more lines. Results showed a PFS of 5.4 months and an OS of 12.2 months, without new adverse effects and being well tolerated. Authors explained these prolonged survivals hypothesizing that previous chemotherapy regimens could induce alterations in intracellular signalling pathways that amplified the reception and efficacy of antiangiogenic drugs.

As we say above, research of "big data" in case of metastatic appendiceal adenocarcinoma is complex, and usually we extrapolate guidelines from metastatic colorectal cancer, when FOLFOX and FOLFIRI (with or without other compounds) are the most common regimens in first- and second-line treatment. However, several authors have explored this field. Asare et al. [12] analyzed a large database of 11,871 patients with appendiceal neoplasms, showing significantly better survival for stage IV mucinous histology versus non-mucinous histology in both subgroups of well-differentiated (6.4 vs. 2.3 years) and poorly differentiated patients (1.5 vs. 0.8 years). Nevertheless, only non-mucinous tumours achieved a significant OS increase with chemotherapy (HR 0.73). Also, histology seemed to play an important role in chemotherapy activity: moderately and poorly differentiated stage IV tumours improved OS with chemotherapy, but not in case of well-differentiated histology. A retrospective review of 112 patients in the NCCN Database, with a majority with non-mucinous histology (48\%) and peritoneum and liver metastatic disease ( 90 and 18\%, respectively), showed median PFS of 1.2 years (95\% CI, 1.0-1.8) and median OS of 2.1 years $(95 \% \mathrm{CI}, 1.6-2.3)$, identifying poor-differentiated histology and non-debulking surgery as worse prognosis factors. No clear benefit when using bevacizumab was observed in this 
report, with a trend towards longer OS in non-bevacizumab regimens $(p=0.41)$ and significant results in PFS favouring bevacizumab-free regimens $(p=0.01)$ [13]. A recent retrospective review of 639 patients with well-differentiated metastatic appendiceal adenocarcinoma achieved significant results in terms of OS for patients who underwent initial surgery (HR 0.40, 95\% CI 0.28-0.57) and also for female sex, but not in the case of patients receiving chemotherapy (HR $1.1,95 \%$ CI $0.56-0.94)$, probably related to a slow cell growth with less chemotherapy activity in this subgroup [14]. A non-significant trend towards an increase in 5-year OS was observed for chemotherapy versus observation (61.3 vs. $52.9 \%$, respectively).

Recent literature has hypothesized that the intense expression of VEGF demonstrated in appendix mucinous adenocarcinoma could be a predictive marker of survival. Logan-Collins et al. [15] demonstrated a correlation between high VEGF levels and poor survival (24.9 vs. 14.7 months, $p=0.017$ ) but not in case of recurrence, so a target treatment against VEGF could theoretically increase tumour sensitivity to anti-angiogenic drugs, which is correlated with high-response rates and survival. Different targetable mutations have also been studied in appendiceal adenocarcinoma, as highly expressed COX-2 (61\%) and KRAS (55\%) in a retrospective study, but no clinical significance or significance for prognosis was observed [16].

To our knowledge, this is the first described case of a patient with a long survival (over 10 years) with stable disease achieved with third-line treatment with capecitabine and bevacizumab, with the peculiarity of achieving a minor response on the three occasions when bevacizumab was reintroduced (having been suspended in a stable-disease situation). We support the hypothesis of the high presence of some unidentified biomarker predictor of response for anti-VEGF therapy in tumour tissue, due to the prolongation of the response over the years and radiological progression of the disease at the time of suspension of bevacizumab. Probably we face a tumour subtype with some unidentified molecular characteristics, making it highly sensitive for this type of drug. Unfortunately, these results are not common for this disease, even less in the case of several previously failed lines of treatment. Therefore, it seems very important to define the biology and the most prevalent targeting molecular alterations, as well as to encourage multidisciplinary collaboration and research of data so as to be able to start prospective trials of appendix malignancies. We hope that this clinical case helps in the search for the best therapy of choice, looking for individualized treatment and the maximum clinical benefit in this malignant disease.

\section{Acknowledgement}

The authors would like to thank all members of the study team, the patient and his family.

\section{Statement of Ethics}

The treatment plan was approved by the Galician Local Research Ethics Committees (GGC-NIV-2018-01) and was executed in accordance with the World Medical Association Declaration of Helsinki, Good Clinical Practice, and local ethical and legal requirements. We obtained informed written consent from the patient authorizing the publication of his clinical case, which is attached to the medical records.

\section{Disclosure Statement}

The authors have no conflicts of interest to declare. 
Arias Ron et al.: Sustained Stable Disease in Appendiceal Adenocarcinoma

\section{Funding Sources}

The study was supported by the Fundación Biomédica Galicia Sur.

\section{Author Contributions}

All authors reviewed the medical records and data and participated in the writing and reviewing of the paper.

\section{References}

1 Marmor S, Portschy PR, Tuttle TM, Virnig BA. The rise in appendiceal cancer incidence: 2000-2009. J Gastrointest Surg. 2015 Apr;19(4):743-50.

2 van den Heuvel MG, Lemmens VE, Verhoeven RH, de Hingh IH. The incidence of mucinous appendiceal malignancies: a population-based study. Int J Colorectal Dis. 2013 Sep;28(9):1307-10.

3 Ito H, Osteen RT, Bleday R, Zinner MJ, Ashley SW, Whang EE. Appendiceal adenocarcinoma: long-term outcomes after surgical therapy. Dis Colon Rectum. 2004 Apr;47(4):474-80.

4 Benson AB, Venook AP, Al-Hawary MM, Cederquist L, Chen YJ, Ciombor KK, et al. NCCN Guidelines Insights: Colon Cancer, Version 2.2018. J Natl Compr Canc Netw. 2018 Apr;16(4):359-69.

5 Peeters M, Price TJ, Cervantes A, Sobrero AF, Ducreux M, Hotko Y, et al. Randomized phase III study of panitumumab with fluorouracil, leucovorin, and irinotecan (FOLFIRI) compared with FOLFIRI alone as second-line treatment in patients with metastatic colorectal cancer. J Clin Oncol. 2010 Nov 1;28(31):4706-13.

6 Saltz LB, Clarke S, Díaz-Rubio E, Scheithauer W, Figer A, Wong R, et al. Bevacizumab in combination with oxaliplatin-based chemotherapy as first-line therapy in metastatic colorectal cancer: a randomized phase III study. J Clin Oncol. 2008 Apr 20;26(12):2013-9.

7 Giantonio BJ, Catalano PJ, Meropol NJ, O'Dwyer PJ, Mitchell EP, Alberts SR, et al. Bevacizumab in combination with oxaliplatin, fluorouracil, and leucovorin (FOLFOX4) for previously treated metastatic colorectal cancer: results from the Eastern Cooperative Oncology Group Study E3200. J Clin Oncol. 2007;25:1539-44.

8 Qu CY, Zheng Y, Zhou M, Zhang Y, Shen F, Cao J, et al. Value of bevacizumab in treatment of colorectal cancer: a meta-analysis. World J Gastroenterol. 2015 Apr 28;21(16):5072-80.

9 Yang Q, Yin C, Liao F, Huang Y, He W, Jiang C, et al. Bevacizumab plus chemotherapy as third- or later-line therapy in patients with heavily treated metastatic colorectal cancer. Onco Targets Ther. 2015 Sep 1;8:240713.

10 Simkens LH, van Tinteren H, May A, ten Tije AJ, Creemers GJ, Loosveld OJ, et al. Maintenance treatment with capecitabine and bevacizumab in metastatic colorectal cancer (CAIR03): a phase 3 randomised controlled trial of the Dutch Colorectal Cancer Group. Lancet. 2015 May;385(9980):1843-52.

11 Larsen FO, Boisen MK, Fromm AL, Jensen BV. Capecitabine and bevacizumab in heavily pre-treated patients with advanced colorectal cancer. Acta Oncol. 2012 Feb;51(2):231-3.

12 Asare EA, Compton CC, Hanna NN, Kosinski LA, Washington MK, Kakar S, et al. The impact of stage, grade, and mucinous histology on the efficacy of systemic chemotherapy in adenocarcinomas of the appendix: analysis of the National Cancer Data Base. Cancer. 2016 Jan;122(2):213-21.

13 Tejani MA, ter Veer A, Milne D, Ottesen R, Bekaii-Saab T, Benson AB 3rd, et al. Systemic therapy for advanced appendiceal adenocarcinoma: an analysis from the NCCN Oncology Outcomes Database for colorectal cancer. J Natl Compr Canc Netw. 2014 Aug;12(8):1123-30.

14 Lu P, Fields AC, Meyerhardt JA, Davids JS, Shabat G, Bleday R, et al. Systemic chemotherapy and survival in patients with metastatic low-grade appendiceal mucinous adenocarcinoma. J Surg Oncol. 2019 Sep;120(3): $446-51$.

15 Logan-Collins JM, Lowy AM, Robinson-Smith TM, Kumar S, Sussman JJ, James LE, et al. VEGF expression predicts survival in patients with peritoneal surface metastases from mucinous adenocarcinoma of the appendix and colon. Ann Surg Oncol. 2008 Mar;15(3):738-44.

16 Raghav KP, Shetty AV, Kazmi SM, Zhang N, Morris J, Taggart M, et al. Impact of molecular alterations and targeted therapy in appendiceal adenocarcinomas. Oncologist. 2013;18(12):1270-7. 